\title{
Stellungnahme zum Kommentar von Sven Rumohr zum Beitrag „Numerische Simulation des Ausbreitungsverhaltens von Wärmeträgerfluid-Inhaltsstoffen aus Erdwärmesonden in Trinkwassereinzugsgebieten“ in Grundwasser 25 (3), 189-204 (2020)
}

\author{
Andreas Dahmke ${ }^{1}$ Dirk Schäfer ${ }^{1} \cdot$ Ralf Köber $^{1} \cdot$ Janine Struß ${ }^{1}$ (D) \\ Eingegangen: 26. November 2020 / Überarbeitet: 4. Dezember 2020 / Angenommen: 10. Dezember 2020 / Online publiziert: 22. Dezember 2020 \\ (C) Der/die Autor(en) 2020
}

Dass die von uns angeregte Diskussion zu Abständen von Erdwärmesonden-Anlagen zu Trinkwasserbrunnen von Herrn Rumohr (Rumohr 2021) aufgegriffen wurde, begrüßen wir sehr und möchten zu seinen Punkten wie folgt Stellung beziehen.

1. Der Schwerpunkt unseres Artikels ist eine numerische und generische Szenarienanalyse zur Bewertung der Sicherheitsabstände von Erdwärmesonden (EWS) bei Leckagefällen zu Trinkwasserbrunnen. Bei allen bekannten intrinsischen und uns auch bewussten Defiziten solcher Szenarienanalysen bieten sie die Möglichkeit, Gefährdungen jedenfalls semiquantitativ abzuschätzen und eine Orientierung bei der Bewertung von Gefährdungen und Risiken von EWS im nicht-bestimmungsgemäßen Betrieb zu geben. Das zusammengefasste Ergebnis der Studie ist, dass die Auswirkungen von EWSLeckagen heute mit gängigen numerischen Ansätzen simuliert werden können und danach die geltenden Sicherheitsabstände bezogen auf die einzelnen Stoffklassen in den Kältemitteln hoch bis sehr hoch sind. Mittelbar geben die Ergebnisse damit auch eine erste, sicherlich noch um empirische Daten zu ergänzende Grundlage, um mögliche EWS-Leckagen vor dem Hintergrund des „Besorgnisgrundsatzes“ faktenbasiert im Vollzug zu bewerten. Dieses ist allerdings nicht die prioritäre Aufgabe von Hochschulen.

2. Uns ist bewusst, dass die Regelungen zum Mindestabstand von EWS-Systemen zu Trinkwasserbrunnen nicht

Janine Struß

janine.struss@ifg.uni-kiel.de

1 Institut für Geowissenschaften,

Christian-Albrechts-Universität zu Kiel,

Ludewig-Meyn-Str. 10, 24118 Kiel, Deutschland allein durch die EWS-Leckagerisiken determiniert sein sollten. So ist es unstrittig, dass unsachgemäß durchgeführte Bohrungen Grundwasser-Gefährdungen, z.B. durch die Schaffung vertikaler Wegsamkeiten zwischen zwei Grundwasserstockwerken, induzieren können. Allerdings existieren nach unserer Kenntnis, mit Ausnahme weniger wertvoller und in Struß et al. (2020) zitierten Studien wie z.B. Grimm et al. (2014), national dazu kaum belastbare und statistisch quantifizierende empirische Evidenzen. Vielmehr beschränkt sich das Wissen darüber i.d.R. auf Fallbeispiele im Sinne zutreffender aber eben auch anekdotischer Berichte. Numerische Simulationen helfen bei dieser Problemstellung zudem nur bedingt weiter, da national einfach die Datenbasis fehlt und weder Häufigkeit (z.B. im Sinne eines statistischen Mittelwertes unsachgemäß durchgeführter Bohrungen) noch deren quantitativen Auswirkungen (z.B. im Sinne induzierter vertikaler Stoffflüsse) derzeit bisher hinreichend statistisch belastbar abgeschätzt wurden. Falls der Kommentar von Herrn Rumohr so verstanden werden darf, dass er hier auch neben einer verbesserten Qualitätssicherung bei Bohrungen ebenso einen relevanten empirisch-experimentellen und geostatistischen Forschungsbedarf sieht, um Abstandsregelungen auf dieser Basis transparent abzuleiten, würden wir ihm beipflichten.

3. Aus übergeordneter Perspektive und in Ergänzung zu den oben skizzierten fachlichen Punkten können wir die Argumentation im Kommentar von Herrn Rumohr bzgl. unserer Aussagen zur Pauschalität und z.T. fehlenden naturwissenschaftlichen Untersetzung von Abstandsregelungen nicht vollständig nachvollziehen. Sowohl die Trinkwasserversorgung als auch eine klimaneutrale, sichere und sozialverträgliche Wärmeversorgung (Heizen \& Kühlen) stellen prioritäre Aufgaben zur Sicherung des Allgemeinwohls dar. Wir sehen daher zukünftig die 
dringliche Notwendigkeit, potenzielle Nutzungskonkurrenzen im untertägigen Raum sachgerecht, großräumig, zeitlich dynamisch und methodisch transparent dreidimensional abzuleiten, zu erfassen und zu bewerten.

Dies gilt insbesondere für urbane Räume mit hohen Bedarfsdichten bei der Wasser-/Trinkwasser- und Wärmeversorgung.

Einfache Kreise mit einem bestimmten Durchmesser um Trinkwasserbrunnen, unabhängig von Strömungsgeschwindigkeit bzw. -richtung und/oder Entnahmerate beispielsweise in den geologischen Untergrund zu projizieren, z.B. zur Festlegung von Restriktionsräumen für EWS-Anlagen, entspricht diesen Anforderungen dort eben nicht mehr und kann eine optimierte Nutzung des geologischen Untergrundes im Sinne des Allgemeinwohls behindern. Ebenso dürfen derzeit sicherlich bestehende und im Kommentar auch beschriebene Defizite beim Grundwassermonitoring bzw. eventuelle personelle Minderausstattungen im Vollzug nicht dazu führen, dass die Umsetzung prioritärer Interessen des Allgemeinwohls im Hinblick auf das Grundwasser per se als nicht umsetzbar angesehen werden.

Ein aus Klimaschutzgründen und zur Sicherung einer sozialverträglichen Wärmeversorgung wahrscheinlich unabdingbarer massiver Ausbau von oberflächennahen thermischen Entzug- und Speichersystemen in urbanen Gebieten wird nur zu realisieren sein, wenn die Nutzung des Grundwassers bzw. des untertägigen Raumes zur Wärme- und Wasserversorgung mit gekoppelten thermisch-hydraulischen Simulationswerkzeugen zukünftig geplant und begleitet wird. In diesem Fall wäre dann die Festlegung von Mindestabständen zwischen Bohrungen und Trinkwasserbrunnen eine ortsspezifische Entscheidung der zuständigen Behörden auf Basis entsprechender numerischer Simulationswerkzeuge. $\mathrm{Zu}$ berücksichtigen wären dabei seitens der Wasserbehörden auch eine übergeordnete kommunenspezifische langfristige Prioritätensetzung bei der Nutzung des oberflächennahen untertägigen Raumes bzw. einzelner Teilräume.

Unbestritten wird dies den Einsatz beträchtlicher und derzeit möglicherweise noch nicht vorhandener finanzieller Mittel z.B. zur Erarbeitung und Weiterentwicklung belastbarer geologischer und hydrogeologischer 3-DModelle erfordern. Aber gerade die Transformation der (urbanen) Wärmeversorgungssysteme bietet auch die wirtschaftliche und regulative Chance, den unverzichtba- ren Grundwasserschutz wieder so auszustatten, dass im Rahmen einer unterirdischen Raumplanung alle Interessen einer nachhaltigen Nutzung des geologischen Untergrundes mit heute zur Verfügung stehenden Methoden optimiert realisiert werden können. Dies einzufordern und umzusetzen sollte nicht zuletzt vor den weiteren Herausforderungen des Klimawandels und den damit einhergehenden Adaptionsnotwendigkeiten gleichermaBen Aufgabe von Praxis und Forschung im Bereich der Hydrogeologie sein.

Funding Open Access funding enabled and organized by Projekt DEAL.

Open Access Dieser Artikel wird unter der Creative Commons Namensnennung 4.0 International Lizenz veröffentlicht, welche die Nutzung, Vervielfältigung, Bearbeitung, Verbreitung und Wiedergabe in jeglichem Medium und Format erlaubt, sofern Sie den/die ursprünglichen Autor(en) und die Quelle ordnungsgemäß nennen, einen Link zur Creative Commons Lizenz beifügen und angeben, ob Änderungen vorgenommen wurden.

Die in diesem Artikel enthaltenen Bilder und sonstiges Drittmaterial unterliegen ebenfalls der genannten Creative Commons Lizenz, sofern sich aus der Abbildungslegende nichts anderes ergibt. Sofern das betreffende Material nicht unter der genannten Creative Commons Lizenz steht und die betreffende Handlung nicht nach gesetzlichen Vorschriften erlaubt ist, ist für die oben aufgeführten Weiterverwendungen des Materials die Einwilligung des jeweiligen Rechteinhabers einzuholen.

Weitere Details zur Lizenz entnehmen Sie bitte der Lizenzinformation auf http://creativecommons.org/licenses/by/4.0/deed.de.

\section{Literatur}

Grimm, M., Stober, I., Kohl, T., Blum, P.: Damage event analysis of drilling borehole heat exchangers 563 in Baden-Württemberg, Germany. Grundwasser 19(4), 275-286 (2014)

Rumohr, S.: Kommentar zum Beitrag „Numerische Simulation des Ausbreitungsverhaltens von Wärmeträgerfluid-Inhaltsstoffen aus Erdwärmesonden in Trinkwassereinzugsgebieten“. Grundwasser - Zeitschrift der Fachsektion Hydrogeologie 26(1), https:// doi.org/10.1007/s00767-020-00468-6 (2021)

Struß, J., Schäfer, D., Dahmke, A., Köber, R.: Numerische Simulationen des Ausbreitungsverhaltens von Wärmeträgerfluid-Inhaltsstoffen aus Erdwärmesonden in Trinkwassereinzugsgebieten. Grundwasser - Zeitschrift der Fachsektion Hydrogeologie 25(3), 189-204 (2020)

Hinweis des Verlags Der Verlag bleibt in Hinblick auf geografische Zuordnungen und Gebietsbezeichnungen in veröffentlichten Karten und Institutsadressen neutral. 